\title{
The Risk and Outcomes of Reoperative Tricuspid Valve Replacement Surgery
}

\author{
Cheng Chen, ${ }^{1,2}$ Min Ge, Jiaxin Ye, ${ }^{2}$ Yongqing Cheng, ${ }^{2}$ Tao Chen, ${ }^{2}$ Lichong Lu, ${ }^{2}$ Zhigang Wang, ${ }^{2}$ \\ Dongjin Wang ${ }^{1,2}$
}

${ }^{1}$ Department of Cardiothoracic Surgery, Drum Tower Clinical Medical College of Nanjing Medical University, Nanjing, China; ${ }^{2}$ Department of Cardiothoracic Surgery, Nanjing Drum Tower Hospital, The Affiliated Hospital of Nanjing University Medical School, Nanjing, China

\section{ABSTRACT}

Objectives: Functional tricuspid regurgitation (TR) usually occurs with previous cardiovascular surgery, which causes right-side heart failure and affects patient prognosis. Thus, we aimed to assess the risk and outcomes of isolated tricuspid valve replacement (TVR) after cardiovascular surgery.

Methods: We reviewed our hospital medical records and found 107 patients, who had undergone TVR following cardiovascular surgery from June 2009 to November 2017. Follow up was performed by telephone calls, with a mean follow up of 51 months (one to 120 months). Previous surgical procedures of all patients were recorded, and we compared the differences in baseline and preoperative characteristics between the survival and non-survival groups by univariate analysis. Furthermore, logistic regression analysis was performed to identify the risk factors. The variables with a $P$ value $<.05$ on univariate analysis were entered into a multivariate analysis using stepwise selection.

Results: TVR was performed in 107 patients, including 89 survivors and 18 non-survivors during the follow up. There were 38 male and 69 female patients, and the mean age was 53.55 years. Hospital mortality was $16.8 \%$ (18/107). The APACHE II $(P<.001)$ and mechanical ventilation time $(P=.001)$ were higher in the non-survival group. The values of B-type natriuretic peptide (BNP), total bilirubin (TB), and blood urea nitrogen (BUN) before and after the operation and some preoperative values were different between the two groups $(P<.05)$. The logistic regression analysis showed that APACHE II score, mechanical ventilation time, preoperative albumin, and postoperative TB were risk factors for TVR after cardiovascular surgery.

Conclusions: Reoperation tricuspid valve replacement is associated with high operative mortality. High APACHE II scores, mechanical ventilation time and postoperative TB were associated with increased short-term mortality risk, while high preoperative albumin levels decreased the risk. Positive reoperation for tricuspid valve prosthesis dysfunction can obtain satisfactory therapeutic effects, and survivors could benefit from the surgery.

Received March 26, 2020; received in revised form fune 16, 2020; accepted fune 16, 2020.

Correspondence: Dongjin Wang, Drum Tower Clinical Medical College of Nanjing Medical University, Zhongshan Road 321, Nanjing, China; +8613915980346 (e-mail: dongjinwang_gl@163.com).

\section{INTRODUCTION}

Tricuspid regurgitation (TR) is a common echocardiographic finding and may be detected in more than $80 \%$ of individuals [Shinn 2013]. The major aetiology of TR is considered secondary to aortic valve and mitral valve disease [Dahou 2015]. As the tricuspid valve (TV) undergoes a low and subsequent pressure, it attracts less attention during left-side heart surgery. When patients have less than severe grades of TR, AHA/ACC guidelines only give Class IIa/IIb recommendations for surgical correction [Baumgartner 2017]. However, the functional TR sometimes does not reverse after left-side valve surgery and may continue to progress [Garcia 2011]. As TR causes right-side heart failure, it will significantly affect the prognosis [Izumi 2011]. Medical therapies, such as diuretics, sometimes may be useful. If drugs do not work, we are willing to consider reoperative tricuspid valve surgery after previous cardiovascular surgery [Kilic 2013].

Tricuspid valve surgery has been suggested to be associated with poor outcomes [Itzhaki 2019]. When a reoperative tricuspid valve replacement is performed, the risk of mortality greatly increases [Abu Sham'a 2013; Hamandi 2019; Campelo-Parada 2017]. However, it also should be pointed out that these results have many confounding factors. It is unknown whether these poor outcomes are related to the surgery itself or to the patients' poor overall status [Pfannmuller 2013]. A researcher has reported that with the development of surgery for TR, the mortality has fallen from $15.9 \%$ to 9.9\% since 2009 [Rodriguez-Capitan 2018]. Studies at different centers have reported variable perioperative outcomes because of an insufficient number of patients, different levels of surgery, and different statuses of patients. Thus, the role of reoperative tricuspid valve surgery still is unclear.

Therefore, it is important to explore the factors that contribute to the high mortality rate in order to improve the postoperative clinical outcome in these patients. This research aimed to review our experience with reoperative tricuspid valve replacement after previous cardiovascular surgery and to determine the factors that influence early and late outcomes.

\section{MATERIALS AND METHODS}

\section{Study population}

From June 2009 to November 2017, a total of 107 consecutive patients underwent isolated TVR with previous 
Table 1. Baseline patient characteristics

\begin{tabular}{|c|c|c|c|c|}
\hline Age (years) & $53.55 \pm 12.50$ & $51.65 \pm 12.50$ & $62.94 \pm 7.38$ & $<.001$ \\
\hline Body surface area $\left(\mathrm{m}^{2}\right)$ & $2.66 \pm 0.27$ & $2.66 \pm 0.46$ & $2.61 \pm 0.39$ & .59 \\
\hline Body mass index $\left(\mathrm{kg} / \mathrm{m}^{2}\right)$ & $22.62 \pm 3.69$ & $22.85 \pm 3.81$ & $21.50 \pm 2.92$ & .15 \\
\hline EuroSCORE (\%) & $16.06 \pm 8.13$ & $14.99 \pm 4.99$ & $21.33 \pm 15.72$ & .646 \\
\hline NYHA class III-IV (n \%) & $81 / 75.7$ & $63 / 70.8$ & $18 / 100$ & .007 \\
\hline LVDd (mm) & $48.39 \pm 6.01$ & $48.58 \pm 6.01$ & $47.45 \pm 6.09$ & .47 \\
\hline LVDs (mm) & $34.54 \pm 5.40$ & $32.85 \pm 4.99$ & $36.08 \pm 5.34$ & .02 \\
\hline LAD (mm) & $56.02 \pm 17.71$ & $56.85 \pm 18.78$ & $51.91 \pm 10.45$ & .28 \\
\hline TR grade: Severe $(n \%)$ & $88 / 81.5$ & $69 / 77.5$ & $18 / 100$ & .026 \\
\hline MR grade: Severe (n \%) & $4 / 3.7$ & $3 / 3.4$ & $1 / 5.6$ & .66 \\
\hline AR grade: Severe ( $n$ \%) & $0 / 0$ & $0 / 0$ & $0 / 0$ & 1 \\
\hline Hepatomegaly (n \%) & $53 / 49.5$ & $46 / 51.7$ & $7 / 38.9$ & .32 \\
\hline Ascites ( $\mathrm{n} \%$ ) & $19 / 17.8$ & $14 / 15.7$ & $5 / 27.8$ & .22 \\
\hline Atrial fibrillation ( $\mathrm{n} \%$ ) & $68 / 63.6$ & $56 / 62.9$ & $12 / 66.7$ & .76 \\
\hline Initial tricuspid surgery & $36 / 33.6 \%$ & $31 / 34.8 \%$ & $5 / 27.8 \%$ & .758 \\
\hline
\end{tabular}

NYHA: New York Heart Association; LVDd: left ventricular diastolic diameter; LVDs: left ventricular systolic diameter; RAD: right atrial diameter; LVEF: left ventricular eject fraction; sPAP: pulmonary artery systolic pressure; TR: tricuspid regurgitation; MR: mitral regurgitation; AR: aortic regurgitation; EuroSCORE: European System for Cardiac Operative Risk Evaluation

cardiovascular surgery at Nanjing Drum Tower Hospital, China. The selection criteria were (i) previous cardiovascular surgery with TVR and (ii) patient age $>18$ years. The exclusion criteria were (i) TVR as the first operation and (ii) congenital Ebstein anomaly.

The study was approved by the Ethics Committee of Nanjing Drum Tower Hospital.

\section{Surgery}

The treatment protocols included detailed explanations to patients, and the treatment was decided together by the cardiac surgeons and patients. All patients underwent elective TVR operations strictly based on the indications. The operations were performed through standard median sternotomy or right antero-lateral thoracotomy. Then, central cannulation or peripheral cannulation were chosen, depending on the sternotomy. Cardiopulmonary bypass (CPB) was performed in a conventional manner to moderate systemic hypothermia. Depending on the patients' conditions, the arrested or beating heart technique was performed. Transoesophageal echocardiography was used during the operation to detect air bubbles and valvular regurgitation. The size of the prosthesis mainly depended on the patients' condition and surgeons' judgement.

\section{Data collection}

Clinical data, including preoperative and postoperative information, were extracted from the hospital's electronic medical record system. Hospital mortality was defined as death within 30 days after the operation or during the same hospitalization. Two-dimensional transthoracic echocardiograms were detected by professional echocardiography doctors in the Department of Medical Ultrasound. Transoesophageal echocardiography was used during the operation. The left ventricular ejection fraction was calculated using Simpson's method. In our hospital, standard practice, according to American Society of Echocardiography guideline criteria, was used to assign grade of valvular regurgitation. Follow-up data were obtained from hospital investigations and telephone interviews. Follow up was closed on November 30, 2019, and 101 of 107 patients (94.4\%) completed the protocol, with a mean follow-up period of 55 months (range one to 120 months).

\section{Statistical analysis}

We express continuous variables as the mean \pm standard deviation. A t-test was used for Student's continuous variables that were normally distributed, and a non-parametric 
Table 2. Previous surgical procedures

\begin{tabular}{lccc}
\hline & All patients $(\mathrm{N}=107)$ & Survival Group $(\mathrm{N}=89)$ & Non-Survival $\mathrm{Group}(\mathrm{N}=18)$ \\
\hline TVR & 3 & 26 & 0 \\
MVR & 26 & 17 & 10 \\
MVR+AVR & 27 & 12 & 3 \\
MVR+AVR+TVP & 15 & 13 & 2 \\
MVR+TVP & 15 & 1 & 1 \\
MVR+AVR+CABG & 2 & 7 & 0 \\
MVP+TVP & 7 & 6 & 1 \\
ASDR or VSDR & 7 & 4 & 1 \\
ASDR or VSDR + others & 5 & 4 & \\
\hline
\end{tabular}

TVR: tricuspid valve replacement; MVR: mitral valve replacement; AVR: aortic valve replacement; MVP: mitral valvuloplasty; TVP: tricuspid valvuloplasty; CABG: coronary artery bypass grafting; ASDR: atrial septal defect repair; VSDR: ventricular septal defect repair

Wilcoxon test was performed for non-normally distributed variables between the survival and non-survival groups. The categorical variables were expressed as frequencies and percentages, and we used Fisher's exact test to compare the differences between groups. We performed logistic regression analysis to identify the risk factors for TVR after cardiovascular surgery. The variables with a $P$ value $<.05$ on univariate analysis were entered into multivariate analysis using stepwise selection.

\section{RESULTS}

Baseline characteristics: The comparative results of baseline characteristics between patients who survived and died after TVR are displayed in Table 1 . There were 38 male and 69 female patients in the study; the average age was $53.55 \pm$ 12.50 years. Patients in the non-survival group significantly were older than those in the survival group $(P<.001)$. However, there were no differences in body surface area $\left(\mathrm{m}^{2}\right)$ or body mass index between the two groups.

Among all the patients, six (5.6\%) were diagnosed with diabetes, and the prevalence was higher in the non-survival group $(P=.025)$. However, there were no differences between the prevalence of both hepatomegaly and ascites between the two groups.

In addition, 81 (75.7\%) patients, including $63(70.8 \%)$ in the survival group and all $18(100 \%)$ patients in the nonsurvival group, were assessed as being in NYHA class 3 or 4 , and the non-survival group had a higher percentage $(P<$ $.01)$. Further analysis of cardiac function showed that the mean left ventricular systolic diameter (LVDs) was $32.85 \pm$ $4.99 \mathrm{~mm}$ in the survival group, which was lower than that in the non-survival group $(36.08 \pm 5.34 \mathrm{~mm})(P=.02)$. However, the left ventricular diastolic diameter (LVDd), left atrial diameter (LAD), right atrial diameter (RAD), left ventricular ejection fraction (LVEF), and pulmonary artery systolic pressure (sPAP) estimated by tricuspid regurgitation were not different between the two groups. The assessment of the severity of cardiac valvular showed that $88(81.5 \%)$ patients were assessed as having severe TR, and the percentage of the non-survival group was higher $(P=.025)$. The percentages of severe aortic regurgitation (AR) and mitral regurgitation (MR) in the two groups were not different. In addition, the difference in atrial fibrillation, with $56(62.9 \%)$ in the survival group and $12(66.7 \%)$ in the non-survival group, also was not significant. It is shown that $36(33.6 \%)$ patients underwent tricuspid surgery with their initial operation. However, there was no difference in the percentages of initial tricuspid operation between the two groups.

Previous surgical procedures: In this study, all patients had previous cardiac surgery history, and the majority underwent the joint procedure (Table 2). Among them, there were 86 cases of valve replacement and seven cases of mitral and tricuspid valvuloplasties. Except for those cases, two cases were double valve replacements combined with coronary artery bypass grafting (CABG). In addition, seven patients underwent atrial septal defect repair (ASDR) or ventricular septal defect repair (VSDR), and the other five patients underwent ASDR or VSDR with other operations.

Univariate analysis based on perioperative patient characteristics: To explore the risk factors of tricuspid valve replacement, we performed univariate analysis based on some perioperative characteristics of all patients. APACHE II is a tool widely used to predict mortality, and the score was $12.61 \pm$ 4.15 in the non-survival group and $8.02 \pm 3.66$ in the survival group; the difference was significant $(P=.001)$. In this study, for BNP, there were significant differences between the two groups before and after the operation. Before the operation, the value of BNP was $128.33 \pm 129.21 \mathrm{pg} / \mathrm{ml}$ in the survival group and $391.21 \pm 376.95 \mathrm{pg} / \mathrm{ml}$ in the non-survival group $(P$ $<.001)$. After the operation, the values were 713.21 \pm 653.50 $\mathrm{pg} / \mathrm{ml}$ and $1470.33 \pm 1468.00 \mathrm{pg} / \mathrm{ml}$, respectively $(P=.003)$.

We found that the mechanical ventilation time in the non-survival group was much longer than that in the survival group, and the mechanical ventilation times were $36.10 \pm$ 
Table 3. Perioperative patient characteristics

\begin{tabular}{|c|c|c|c|c|}
\hline CPB time (min) & $163.73 \pm 68.91$ & $157.98 \pm 52.65$ & $192.17 \pm 119.30$ & .270 \\
\hline Bioprosthetic valve: Mechanical valve & $25: 82$ & $22: 67$ & $3: 15$ & .462 \\
\hline Median sternotomy: Right thoracotomy & $26: 81$ & $21: 68$ & $5: 13$ & .520 \\
\hline Mechanical ventilation time $(h)$ & $70.08 \pm 155.15$ & $36.10 \pm 37.29$ & $238.10 \pm 327.04$ & .001 \\
\hline pre-ALT $(U / L)$ & $17.34 \pm 6.35$ & $17.42 \pm 6.68$ & $16.99 \pm 4.50$ & .927 \\
\hline pre-TB (mmol/L) & $24.69 \pm 15.80$ & $22.54 \pm 12.53$ & $35.31 \pm 24.52$ & .017 \\
\hline pre-Albumin (g/L) & $39.21 \pm 5.49$ & $40.17 \pm 5.21$ & $34.43 \pm 4.29$ & .000 \\
\hline pre-BUN (mmol/L) & $7.64 \pm 3.59$ & $6.99 \pm 2.69$ & $10.88 \pm 5.46$ & .000 \\
\hline post-ALT $(\mathrm{U} / \mathrm{L})$ & $28.5944 \pm 16.65$ & $27.82 \pm 17.78$ & $32.41 \pm 20.50$ & .777 \\
\hline post-TB (mmol/L) & $52.615 \pm 47.13$ & $41.94 \pm 33.60$ & $105.38 \pm 66.82$ & .000 \\
\hline post-Albumin $(\mathrm{g} / \mathrm{L})$ & $39.1785 \pm 15.39$ & $37.86 \pm 6.00$ & $45.70 \pm 35.17$ & .464 \\
\hline post-BUN (mmol/L) & $15.166 \pm 45.96$ & $9.569 \pm 3.43$ & $44.47 \pm 112.74$ & .000 \\
\hline post-Scr $(\mu \mathrm{mol} / \mathrm{L})$ & $96.6321 \pm 37.91$ & $97.01 \pm 39.42$ & $94.64 \pm 29.62$ & .763 \\
\hline post-BNP $(\mathrm{pg} / \mathrm{ml})$ & $840.58 \pm 883.82$ & $713.21 \pm 653.50$ & $1470.33 \pm 1468.00$ & .003 \\
\hline ICU stay (h) & $185.07 \pm 237.47$ & $147.38 \pm 134.91$ & $371.4 \pm 461.72$ & .474 \\
\hline Fluid volume (ml) & $-220.21 \pm 682.32$ & $-275.14 \pm 610.59$ & $51.33 \pm 937.67$ & .017 \\
\hline
\end{tabular}

$37.29 \mathrm{~h}$ and $238.10 \pm 327.04 \mathrm{~h}$, respectively $(P=.001)$. The CPB time $(157.98 \pm 52.6 \mathrm{~min}$ in the survival group and 192.17 $\pm 119.30 \mathrm{~min}$ in the non-survival group) and cross clamp time $(94.79 \pm 36.73 \mathrm{~min}$ in the survival group and $83.00 \pm 43.09$ min in the non-survival group) were not significantly different. In addition, we analyzed preoperative and postoperative liver function (alanine transaminase (ALT), TB, albumin), and renal function (BUN, serum creatinine). The values of preoperative TB, albumin, and BUN and the values of postoperative TB and BUN were different between the two groups $(P<.05)$. Moreover, although the time patients stayed in the intensive care unit was not different, the fluid volume was significantly different. The fluid volume on the first day of ICU in the non-survival group was significantly different from that in the survival group $(P=.017)$. When the value was greater than 0 , it meant we had replenished the body with fluid. In contrast, when the value was less than 0 , it meant we had dehydrated the patient.

The logistic regression analysis results of risk factors for death of TVR after cardiovascular surgery are displayed in Table 4. These factors, including mechanical ventilation time (OR 0.984, $P=.019$ ), preoperative albumin (OR $1.201, P=.032)$, postoperative TB (OR 0.935, $P=.002)$, and APACHE II (OR $0.725, P=.007)$, were determined to be risk factors.

\section{DISCUSSION}

The choice for treating TR is conservative therapy, especially after the first cardiovascular surgery. Even when TR is severe, the first approach for patients is medical treatment. When clinical symptoms become unbearable, patients are compelled to accept other therapies, such as reoperation. By this time, right heart failure is very severe and irreversible. Thus, it has been shown that the hospital mortality rate is more than $35 \%$ in patients who undergo TVR after previous TV repair [Bernal 2005]. TVR was the main measure for patients with severe TR after previous tricuspid valve repair and secondary severe TR with left heart valve disease [Guenther 2008; Mao 2016]. However, as the surgical expertise of TVR has rapidly improved in recent years, the mortality has dropped dramatically [Park 2009; Moutakiallah 2018]. In our study, the hospital mortality rate was $16.8 \%(\mathrm{~N}=18)$, which fell in between the values in the above-mentioned studies.

Causes of death were extremely complicated. Pfannmuller et al observed that the mortality would decrease from $35.7 \%$ to $4.0 \%$, when an elective operation rather than a non-elective procedure was performed. The researchers also confirmed that they focused on patients with isolated TV surgery. These patients may be strictly selected [Pfannmuller 2013]. Because, as Buzzatti et al indicated, tricuspid disease after previous 
Table 4. Multivariable analysis of factors affecting hospital mortality

\begin{tabular}{llll}
\hline & $P$ & OR & $95 \% \mathrm{Cl}$ \\
\hline Mechanical ventilation time (h) & .019 & 0.984 & $0.972-0.997$ \\
pre-Albumin (g/L) & .032 & 1.201 & $1.016-1.421$ \\
post-TB (mmol/L) & .002 & 0.935 & $0.896-0.975$ \\
APACHE II & .007 & 0.725 & $0.572-0.917$ \\
\hline
\end{tabular}

TB: total bilirubin; APACHE: Acute Physiology and Chronic Health Evaluation

Supplemental Table 1. Perioperative cardiac ultrasound results of survivals

\begin{tabular}{|c|c|c|c|c|c|}
\hline LVDd & $48.58 \pm 6.01$ & $49.98 \pm 5.36$ & .20 & $52.15 \pm 5.43$ & .005 \\
\hline LAD & $56.85 \pm 18.78$ & $52.84 \pm 15.78$ & .233 & $54.62 \pm 17.17$ & .567 \\
\hline LVEF & $51.90 \% \pm 6.39 \%$ & $53.55 \% \pm 5.34 \%$ & .123 & $53.97 \% \pm 4.68 \%$ & .06 \\
\hline
\end{tabular}

left-side surgery is not an isolated problem, the number of isolated TVRs is low, and patients always require contemporary associated procedures [Buzzatti 2014]. Consistent with this result, we also found that the number of combined procedures was greater than that of isolated TVRs. Moreover, the non-survival group had more underlying diseases, such as diabetes mellitus $(P=.025)$. Age also may be a reason for a bad outcome. We found that the median age of the non-survival group was 10 years older than that of the survival group $(P<$ $.001)$. An older age in patients usually meant advanced stages of the disease and less recovery.

In many studies, NYHA functional class III/IV has been proven to be an independent risk factor for mortality [Chen 2018]. The presence of NYHA functional class III/IV implied that not much was left for a cushion and the heart could not withstand an extra strike. In our study, the NYHA function of all the patients in the non-survival group was grade III/IV, which was significantly higher than the grade in the survival group $(100 \%$ versus $70.8 \%, P=.007)$. Moderate or severe TR also has been shown to have a negative impact on survival [Nath 2004]. Subbotina and his team demonstrated that patients with severe right ventricular dysfunction not only more frequently underwent acute decompensations preoperatively, but also presented with poorer outcomes after a TVR operation [Subbotina 2017]. Similarly with the NYHA functional class, the incidence of severe TR also was 100\% in the non-survival group. Thus, the operation mortality risk was greater when both left and right heart function were severely reduced.

When focusing on the TVR itself and perioperative biochemical indices, we obtained many interesting discoveries. After sternotomy, it is known that the heart would attach to the sternum and the tissue would become more adherent.
Thus, the choice of surgical incision is difficult and crucial. Emerging researchers have preferred right mini-thoracotomy rather than median sternotomy because the former procedure could reduce the separation of adhesive tissues, intraoperative bleeding, and consequent right heart dysfunction [ $\mathrm{Zhu}$ 2018; Fang 2018]. However, we found no difference between the two groups. We thought the incision should be chosen according to the specific situation of the patient, especially with regard to the previous operation. However, the choice between mechanical and biological valve for TVR is controversial [Hwang 2014]. In our hospital, we selected the valve based on the maximum life expectancy of the patients. Some researchers showed no significant differences between mechanical and biologic prostheses, in terms of early and late survival [Pfannmuller 2013; Fender 2018].

In our center, every patient who underwent cardiac surgery was admitted to the intensive care unit (ICU) of our own department. The Acute Physiology and Chronic Health Evaluation II (APACHE II) score is a tool widely used to predict the mortality of patients admitted to the ICU [Nimgaonkar 2004; Pirracchio 2015]. The score was statistically significant between the non-survival group and the survival group $(P<.001)$. It has been proven that $10-20 \%$ of cardiac surgery patients have to prolong postoperative mechanical ventilation and thus comprise a specific group with a high risk of postoperative mortality [FernandezZamora 2018]. Pneumonia, organ failure, and sepsis caused by prolonged mechanical ventilation were the most common reasons associated with increased hospital mortality and reduced long-term survival [Ibanez 2016]. In the use of a ventilator, we try to adopt a protective ventilation strategy and attempt to get the patient off the ventilator as soon as possible. Preoperative optimization of heart failure, including 
liver function and nutritional status, is the consensus before TVR [Pichette 2017]. Through a large retrospective study, Kingeter et al found that compared with the infusion of crystalloids alone, the use of $5 \%$ albumin solution significantly decreased patients' in-hospital mortality and 30-day readmission rate after cardiac surgery [Kingeter 2018]. We also suggest that a high preoperative albumin level is a protective factor (OR 1.201, $P=.032)$.

\section{LIMITATIONS}

This research has some limitations. Our study was a singlecenter retrospective study, and the study population was comparatively small. Due to inherent limitations, some variables were not analyzed. The first surgery before tricuspid valve surgery was complex, which resulted in greater heterogeneity of the enrolled patients. The differences in preoperative and intraoperative echocardiographic evaluations may have led to some errors in the results. Moreover, because of economic or cultural reasons, the patients did not receive a long-term echocardiographic follow up. Consequently, we cannot further evaluate their postoperative tricuspid valve conditions.

\section{CONCLUSION}

In conclusion, we described reoperation for tricuspid valve replacement following cardiac surgery. High APACHE II scores, mechanical ventilation time and postoperative TB were associated with increased short-term mortality risk, while high preoperative albumin levels decreased the risk. There is no doubt that TVR after previous cardiovascular surgery has a high mortality. However, it can significantly improve the quality of life and prolong survival time, so TVR should still be considered.

\section{REFERENCES}

Abu Sham'a R, Buber J, Grupper A, et al. 2013. Effects of tricuspid valve regurgitation on clinical and echocardiographic outcome in patients with cardiac resynchronization therapy. Europace 15:266-72.

Baumgartner H, Falk V, Bax JJ, et al. 2017. 2017 ESC/EACTS Guidelines for the management of valvular heart disease. Eur Heart J 38:2739-91.

Bernal JM, Morales D, Revuelta C, et al. 2005. Reoperations after tricuspid valve repair. J Thorac Cardiovasc Surg 130:498-503.

Buzzatti N, Iaci G, Taramasso M, et al. 2014. Long-term outcomes of tricuspid valve replacement after previous left-side heart surgery. European journal of cardio-thoracic surgery: official journal of the European Association for Cardio-Thoracic Surgery 46:713-9; discussion 9.

Campelo-Parada F, Lairez O, Carrie D. 2017. Percutaneous Treatment of the Tricuspid Valve Disease: New Hope for the "Forgotten" Valve. Rev Esp Cardiol (Engl Ed) 70:856-66.

Chen J, Abudupataer M, Hu K, et al. 2018. Risk factors associated with perioperative morbidity and mortality following isolated tricuspid valve replacement. The Journal of Surgical Research 221:224-31.
Dahou A, Magne J, Clavel MA, et al. 2015. Tricuspid Regurgitation Is Associated With Increased Risk of Mortality in Patients With Low-Flow Low-Gradient Aortic Stenosis and Reduced Ejection Fraction: Results of the Multicenter TOPAS Study (True or Pseudo-Severe Aortic Stenosis). JACC Cardiovasc Interv 8:588-96.

Fang L, Li W, Zhang W, Gu W, Zhu D. 2018. Mid-term results and risks of isolated tricuspid valve reoperation following left-sided valve surgery. European journal of cardio-thoracic surgery: official journal of the European Association for Cardio-Thoracic Surgery 53:1034-9.

Fender EA, Zack CJ, Nishimura RA. 2018. Isolated tricuspid regurgitation: outcomes and therapeutic interventions. Heart 104:798-806.

Fernandez-Zamora MD, Gordillo-Brenes A, Banderas-Bravo E, et al. 2018. Prolonged Mechanical Ventilation as a Predictor of Mortality After Cardiac Surgery. Respir Care 63:550-7.

Garcia Fuster R, Vazquez A, Pelaez AG, et al. 2011. Factors for development of late significant tricuspid regurgitation after mitral valve replacement: the impact of subvalvular preservation. Eur J Cardiothorac Surg 39:866-74; discussion 74 .

Guenther T, Noebauer C, Mazzitelli D, et al. 2008. Tricuspid valve surgery: a thirty-year assessment of early and late outcome. Eur J Cardiothorac Surg 34:402-9; discussion 9.

Hamandi M, Smith RL, Ryan WH, et al. 2019. Outcomes of Isolated Tricuspid Valve Surgery Have Improved in the Modern Era. Ann Thorac Surg 108:11-5.

Hwang HY, Kim KH, Kim KB, Ahn H. 2014. Propensity score matching analysis of mechanical versus bioprosthetic tricuspid valve replacements. Ann Thorac Surg 97:1294-9.

Ibanez J, Riera M, Amezaga R, et al. 2016. Long-Term Mortality After Pneumonia in Cardiac Surgery Patients: A Propensity-Matched Analysis. J Intensive Care Med 31:34-40.

Itzhaki Ben Zadok O, Sagie A, Vaturi M, et al. 2019. Long-Term Outcomes After Mitral Valve Replacement and Tricuspid Annuloplasty in Rheumatic Patients. Ann Thorac Surg 107:539-45.

Izumi C, Miyake M, Takahashi S, et al. 2011. Progression of isolated tricuspid regurgitation late after left-sided valve surgery. Clinical features and mechanisms. Circ J 75:2902-7.

Kilic A, Saha-Chaudhuri P, Rankin JS, Conte JV. 2013. Trends and outcomes of tricuspid valve surgery in North America: an analysis of more than 50,000 patients from the Society of Thoracic Surgeons database. Ann Thorac Surg 96:1546-52; discussion 52.

Kingeter AJ, Raghunathan K, Munson SH, et al. 2018. Association between albumin administration and survival in cardiac surgery: a retrospective cohort study. Can J Anaesth 65:1218-27.

Mao B, Sun L, Zhang J, Zhou M, Zhang F. 2016. Perioperative factors associated with short- and long-term outcomes after tricuspid valve replacement. Interact Cardiovasc Thorac Surg 23:845-50.

Moutakiallah Y, Aithoussa M, Atmani N, et al. 2018. Reoperation for isolated rheumatic tricuspid regurgitation. J Cardiothorac Surg 13:104.

Nath J, Foster E, Heidenreich PA. 2004. Impact of tricuspid regurgitation on long-term survival. Journal of the American College of Cardiology 43:405-9.

Nimgaonkar A, Karnad DR, Sudarshan S, Ohno-Machado L, Kohane I. 2004. Prediction of mortality in an Indian intensive care unit. Comparison between APACHE II and artificial neural networks. Intensive Care Med 30:248-53. 
Park CK, Park PW, Sung K, et al. 2009. Early and midterm outcomes for tricuspid valve surgery after left-sided valve surgery. The Annals of Thoracic Surgery 88:1216-23.

Pfannmuller B, Moz M, Misfeld M, et al. 2013. Isolated tricuspid valve surgery in patients with previous cardiac surgery. The Journal of Thoracic and Cardiovascular Surgery 146:841-7.

Pichette M, Liszkowski M, Ducharme A. 2017. Preoperative Optimization of the Heart Failure Patient Undergoing Cardiac Surgery. Can J Cardiol 33:72-9.

Pirracchio R, Petersen ML, Carone M, et al. 2015. Mortality prediction in intensive care units with the Super ICU Learner Algorithm (SICULA): a population-based study. Lancet Respir Med 3:42-52.

Rodriguez-Capitan J, Becerra-Munoz VM, Perez-Villardon B, et al. 2018. Clinical outcomes after tricuspid surgery: The role of previous cardiac surgery. Herz.

Shinn SH, Schaff HV. 2013. Evidence-based surgical management of acquired tricuspid valve disease. Nat Rev Cardiol 10:190-203.

Subbotina I, Girdauskas E, Bernhardt AM, et al. 2017. Comparison of Outcomes of Tricuspid Valve Surgery in Patients with Reduced and Normal Right Ventricular Function. Thorac Cardiovasc Surg 65:617-25.

Zhu X, Luo Y, Zhang E, et al. 2018. Ten-year experience of tricuspid valve replacement with the St. Jude medical valve. Sci Rep 8:16654. 